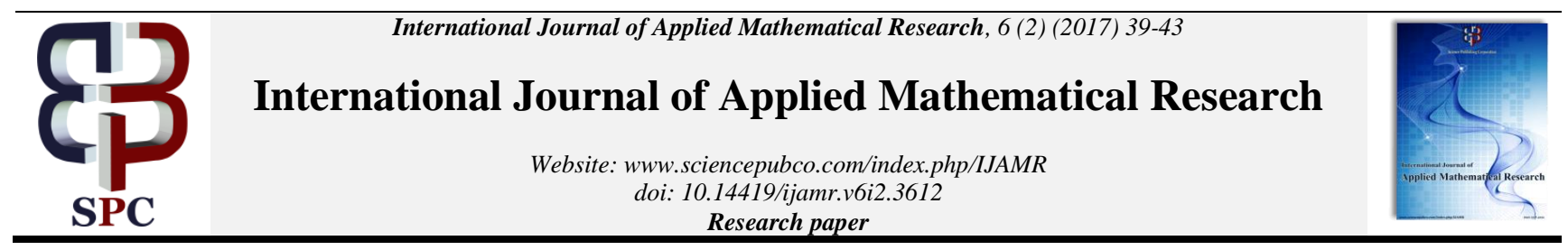

\title{
Early response of soils to violent disturbances
}

\author{
Abdelwahab Kharab *, Jamal Benbourenane \\ Abu Dhabi University, Abu Dhabi, UAE \\ *Corresponding author E-mail: abdelwahab.kharab@adu.ac.ae
}

\begin{abstract}
A one-dimensional model dealing with underground explosions as experienced in areas such as mining or excavations is presented. When an explosion in a typical soil medium occurs, soil material is displaced and shock waves propagate in the soil medium. Soil is considered as a floating, ideally locking material. In this paper, the speed of propagation for the shock waves is analyzed, and results are given. The Mie-Gruneisen equation of state is used to find the pressure as a function of the density. Results with the present model yield an efficient and comprehensive means to analyze speed of waves in a sandy medium.
\end{abstract}

Keywords: Explosion; Shock Waves; Soil; Penetration; Speed.

\section{Introduction}

Over the previous several decades, enormous work has gone into understanding the response of soils to violent disturbances. This work has been motivated by efforts to model underground excavations, penetration of projectiles in targets, and other applications. Unfortunately, most of the work done on projectile penetration has focused on steel, metal and aluminum targets with different thickness such as thick, intermediate and thin plates. The analysis of modern state of the problem of analytical of highvelocity penetration of a projectile in target can be found in works by Forrestal ([3],[4]), Warren and Forrestal [11], Yarin et al. [9], Bachman and Goldsmith [2], Chen and Li [5] and Roisman [10]. However, sufficient data is available so that an equation of state for certain soils can be obtained.

Despite the importance and implications of the subject, little information exists in literature on the mechanisms involved during rigid body penetration into terrestrial materials and underground explosions. The reasons behind the lack of information about the mechanisms' penetration are obvious. The inherent mathematical complexities associated with defining constitutive equations and developing solutions for the conservation equations make the problem very hard to deal with.

Problems dealing with the underground explosion and penetration of a rigid body into a soil medium have many applications in the real word. Examples of such applications are mining, construction, excavations and putting down pilings in soils for large buildings.

This paper presents the results of a numerical study of shock waves that arise from underground explosions as experienced in areas such as mining, excavations and the penetration of soils by rigid bodies. The main purpose of this investigation is to study the speed of shock waves through a soil medium. Two-time intervals are observed after the explosion, the first starts at time $t=0$ of the explosion when the disturbance begins, and the second is when the shock starts to dissipate.

\section{Model problem}

In order to describe the speed of propagation for the shock waves in a sandy medium caused by an underground explosion (see Fig. 1), the following assumption is made:

The medium is assumed to be a sandy medium, deformable and visco-plastic.

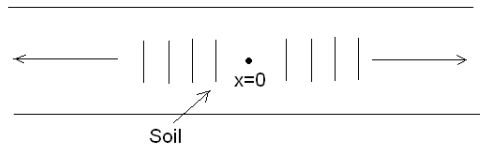

Fig. 1: Listing of the 1-2-3 Macro.

We begin with the following system of partial differential equations based on the laws of conservation of mass, momentum, and energy.

$$
\frac{\partial \rho}{\partial t}+\frac{\partial}{\partial x}(\rho v)=0
$$

$\rho\left[\frac{\partial v}{\partial t}+v \frac{\partial v}{\partial x}\right]=-\frac{\partial p}{\partial x}+\frac{\partial \tau}{\partial x}$

$\rho\left[\frac{\partial \mathrm{e}}{\partial \mathrm{t}}+\mathrm{v} \frac{\partial \mathrm{e}}{\partial \mathrm{x}}\right]=-\mathrm{p} \frac{\partial \mathrm{v}}{\partial \mathrm{x}}$

In this model, $\rho$ represents the mass density of the medium, $\mathrm{v}$ the eulerian velocity field of the medium, e the specific internal energy, i.e. energy/mass and $p$ is the pressure. $\partial \tau / \partial x$ is the deviatoric stress tensor of the form given by Papanastasiou and Boudouvis (1997):

$\tau=2 \alpha(\mathrm{D}) \mathrm{D}$ 
Where $\alpha(D)$ is a scalar function and $D=\frac{\partial v}{\partial x}$, is the rate of deformation tensor. The function $\alpha(D)$ is given by

$\alpha(D)=\mu\left\{1+\frac{\varepsilon}{|D|}[1-\exp (-n|D|)]\right\}, \quad$ ifD $\neq 0$

$\alpha(0)=1+\varepsilon n$,

$\mu$ is the dynamic viscosity of the saturated medium and $\varepsilon$ and $n$ are empirical constants.

We assume the explosion takes place at the time $t=0$ and the position $\mathrm{x}=0$. The following initial conditions for $\mathrm{x} \geq 0$ are used:

$\rho(x, 0)=\rho_{0} \mathrm{~g} / \mathrm{cm}^{3}$,

$\mathrm{v}(\mathrm{x}, 0)=\delta(\mathrm{x}) \mathrm{cm} / \mathrm{sec}$,

$\mathrm{e}(\mathrm{x}, 0)=\mathrm{e}_{0} \mathrm{~J} / \mathrm{kg}$

Where $\delta(\mathrm{x})$ is the delta function.

\section{Pressure model in sand}

The shock pressure in the sandy medium is assumed to be governed by the Mie-Gruneisen equation of state, given by

$$
\mathrm{p}(\rho, \mathrm{e})=\frac{\gamma_{\mathrm{o}} \rho_{0}}{1+\gamma_{\mathrm{o}} \eta} \mathrm{e}+\rho_{0} \mathrm{C}_{0}^{2} \eta \frac{2-\gamma_{\mathrm{o}} \eta}{2\left(1+\gamma_{\mathrm{o}} \eta\right)(1-\mathrm{s} \eta)^{2}}
$$

Where $\eta=1-\rho_{0} / \rho, \gamma_{0}$ is the Gruneisen parameter (unitless), $s$ is a positive number (unitless) and $\mathrm{C}_{0}$ is the dimensional reference sound speed for the target medium.

If the impact shock occurs so rapidly, then the heat conduction becomes negligible and; consequently, by using Gibb's relation, the Mie-Gruneisen equation of state can be written in the form

$\mathrm{p}(\rho)=\gamma_{\mathrm{o}} \rho_{0} \mathrm{e}_{0}+\frac{1}{2} \gamma_{\mathrm{o}} \rho_{0} \mathrm{c}_{0}^{2}\left[\begin{array}{l}\mathrm{A} \ln \left(1+\gamma_{\mathrm{o}} \eta\right) \\ +\mathrm{B} \frac{\eta}{1+\gamma_{\mathrm{o}} \eta} \\ -\mathrm{A} \ln (1-\mathrm{s} \eta) \\ +\mathrm{C} \frac{\eta}{1-\mathrm{s} \eta}\end{array}\right]$

$$
\begin{aligned}
& +\frac{\rho_{0} \mathrm{c}_{0}^{2} \eta\left(2+\gamma_{\mathrm{o}} \eta\right)}{2\left(1+\gamma_{\mathrm{o}} \eta\right)(1-s \eta)^{2}}, \\
& \mathrm{~A}=\frac{-2 \mathrm{~s}}{\left(\gamma_{\mathrm{o}}+\mathrm{s}\right)^{3}}, \quad \mathrm{~B}=\frac{-\gamma_{0}}{\left(\gamma_{\mathrm{o}}+\mathrm{s}\right)^{2}}, \\
& \mathrm{C}=\frac{\gamma_{\mathrm{o}}+2 \mathrm{~s}}{\left(\gamma_{\mathrm{o}}+s\right)^{2}}, \quad \eta=1-\frac{\rho_{0}}{\rho}
\end{aligned}
$$

Which is referred as the Hugoniot-adiabat.

\section{Time intervals}

There are two times intervals that are identified following an underground explosion. The first time interval is the elapsed time in which the deviatoric stress tensor has no effect (see [7]) and thus near $\mathrm{x}=0$, one can see the material rushing out. The second time interval starts when the deviatoric stress tensor become important and is no more negligible.

If the impact shock occurs rapidly, then the heat conduction becomes negligible and; consequently during the first time interval a scaling argument shows that the contribution of the deviatoric stress tensor is negligible and so only the first two equations, (1), (2), with the Hugoniot-adiabat equation (7) enter into the calculations. After $0.001 \mathrm{msec}$., time at which the second interval starts, the full system of equations (1-3) with the Gruneisen equation of state (6) will used to compute the speed.

\section{Numerical solution}

The Lax-Wendroff scheme will be used to find the numerical solution to our model problem which requires that the differential equations be written in a conservative form $\frac{\partial u}{\partial t}=-\frac{\partial j}{\partial x}$, so the original system (1)-(3) is written as follows:

$\frac{\partial \rho}{\partial t}=-\frac{\partial}{\partial x}(\rho v)$

$\frac{\partial}{\partial t}(\rho v)=-\frac{\partial}{\partial x}\left[\frac{(\rho v)^{2}}{\rho}+p-\tau\right]$

$\frac{\partial}{\partial t}\left[\rho\left(e+\frac{1}{2} v^{2}\right)\right]=-\frac{\partial}{\partial x}\left[\rho v\left(e+\frac{1}{2} v^{2}\right)+v p\right]$

Let $w=\rho v$ and $E=\rho\left(e+\frac{1}{2} v^{2}\right)$ then the system (8) can be written as

$\frac{\partial \rho}{\partial t}=-\frac{\partial w}{\partial x}$

$\frac{\partial w}{\partial t}=-\frac{\partial}{\partial x}\left(\frac{w^{2}}{\rho}+p-\tau\right)$

$\frac{\partial \mathrm{E}}{\partial \mathrm{t}}=-\frac{\partial}{\partial \mathrm{x}}\left[\frac{\mathrm{w}}{\rho}(\mathrm{E}+\mathrm{p})\right]$

For initial value problems involving a conservative-hyperbolic differential equation of the form $\frac{\partial u}{\partial t}=-\frac{\partial j}{\partial x}$, the Lax-Wendroff scheme employs the leapfog method with half-step to calculate new values for $u$ at each time step. That is, let $\rho_{i}^{n}=\rho(i h, n k)$, $w_{i}^{n}=w(i h, n k)$ and $E_{i}^{n}=E(i h, n k), h, k$, being the mesh spacing in the space and time directions respectively. We have:

At the half time grid $t=t_{i+1 / 2}$ and half time space $x=x+1 / 2$

$\rho_{\mathrm{i}+1 / 2}^{\mathrm{n}+1 / 2}=\frac{\rho_{\mathrm{i}+1}^{\mathrm{n}}+\rho_{\mathrm{i}}^{\mathrm{n}}}{2}-\frac{1}{2} \frac{\mathrm{k}}{\mathrm{h}}\left(\mathrm{w}_{\mathrm{i}+1}^{\mathrm{n}}-\mathrm{w}_{\mathrm{i}}^{\mathrm{n}}\right)$

$w_{i+1 / 2}^{n+1 / 2}=\frac{w_{i+1}^{n}+w_{i}^{n}}{2}$

$-\frac{1}{2} \frac{k}{h}\left\{\left[\begin{array}{l}\frac{\left(w_{i+1}^{n}\right)^{2}}{\rho_{i+1}^{n}} \\ +p_{i+1}^{n}-\tau_{i+1}^{n}\end{array}\right]-\left[\begin{array}{c}\frac{\left(w_{i}^{n}\right)^{2}}{\rho_{i}^{n}} \\ +p_{i}^{n}-\tau_{i}^{n}\end{array}\right]\right\}$ 


$$
\begin{aligned}
& E_{i+1 / 2}^{n+1 / 2}=\frac{E_{i+1}^{n}+E_{i}^{n}}{2} \\
& -\frac{1}{2} \frac{k}{h}\left[w_{i+1}^{n}\left(E_{i+1}^{n}+p_{i+1}^{n}\right) / \rho_{i+1}^{n}-w_{i}^{n}\left(E_{i}^{n}+p_{i}^{n}\right) / \rho_{i}^{n}\right] \\
& \tau_{i}^{n}=2 \alpha\left(D_{i}^{n}\right) D_{i}^{n}, \\
& D_{i}^{n}=\left(v_{i+1}^{n}-v_{i}^{n}\right) / h=\left(w_{i+1}^{n}-w_{i}^{n}\right) /\left(h \rho_{i}^{n}\right) \\
& \text { at } \mathrm{t}=\mathrm{t}_{\mathrm{i}+1} \\
& \rho_{i}^{n+1}=\rho_{i}^{n}-\frac{k}{h}\left(w_{i+1 / 2}^{n+1 / 2}-w_{i-1 / 2}^{n+1 / 2}\right) \\
& w_{i}^{n+1}=w_{i}^{n} \\
& -\frac{\mathrm{k}}{\mathrm{h}}\left\{\left[\frac{\left(\mathrm{w}_{\mathrm{i}+1 / 2}^{\mathrm{n}+1 / 2}\right)^{2}}{\rho_{\mathrm{i}+1 / 2}^{\mathrm{n}+1 / 2}}+\mathrm{p}_{\mathrm{i}+1 / 2}^{\mathrm{n}+1 / 2}-\tau_{\mathrm{i}+1 / 2}^{\mathrm{n}+1 / 2}\right]-\left[\frac{\left(\mathrm{w}_{\mathrm{i}-1 / 2}^{\mathrm{n}+1 / 2}\right)^{2}}{\rho_{\mathrm{i}-1 / 2}^{\mathrm{n}+1 / 2}}+\mathrm{p}_{\mathrm{i}-1 / 2}^{\mathrm{n}+1 / 2}-\tau_{\mathrm{i}-1 / 2}^{\mathrm{n}+1 / 2}\right]\right\} \\
& \mathrm{E}_{\mathrm{i}}^{\mathrm{n}+1}=\mathrm{E}_{\mathrm{i}}^{\mathrm{n}} \\
& -\frac{k}{h}\left[\begin{array}{c}
w_{i+1 / 2}^{n+1 / 2}\left(E_{i+1 / 2}^{n+1 / 2}+p_{i+1 / 2}^{n+1 / 2}\right) / \rho_{i+1 / 2}^{n+1 / 2} \\
-w_{i-1 / 2}^{n+1 / 2}\left(E_{i-1 / 2}^{n+1 / 2}+p_{i-1 / 2}^{n+1 / 2}\right) / \rho_{i-1 / 2}^{n+1 / 2}
\end{array}\right]
\end{aligned}
$$

where in the first time interval $\mathrm{p}$ is given by

$$
\begin{aligned}
& \mathrm{p}_{i}^{\mathrm{n}}=\mathrm{p}\left(\rho_{i}^{\mathrm{n}}\right)=\gamma_{\mathrm{o}} \rho \rho^{\mathrm{e}} 0 \\
& +\frac{1}{2} \gamma_{o} \rho_{0} c_{0}^{2}\left[\begin{array}{l}
A \ln \left(1+\gamma_{o} \eta_{i}^{n}\right) \\
+B \frac{\eta_{i}^{n}}{1+\gamma_{o} \eta_{i}^{n}}-A \ln \left(1-s \eta_{i}^{n}\right) \\
+C \frac{\eta_{i}^{n}}{1-s \eta_{i}^{n}}
\end{array}\right] \\
& +\frac{\rho_{0} c_{0}^{2} \eta_{i}^{n}\left(2+\gamma_{o} \eta_{i}^{n}\right)}{2\left(1+\gamma_{o} \eta_{i}^{n}\right)\left(1-s \eta_{i}^{n}\right)^{2}} \\
& \eta_{i}^{n}=1-\frac{\rho_{0}}{\rho_{i}^{n}}, \quad A=\frac{-2 s}{\left(\gamma_{0}+s\right)^{3}}, \\
& B=\frac{-\gamma_{o}}{\left(\gamma_{o}+s\right)^{2}}, \quad C=\frac{\gamma_{o}+2 s}{\left(\gamma_{o}+s\right)^{2}},
\end{aligned}
$$

and in the second time interval

$$
\begin{aligned}
& \mathrm{p}_{\mathrm{i}}^{\mathrm{n}}=\mathrm{p}\left(\rho_{\mathrm{i}}^{\mathrm{n}}, \mathrm{e}_{\mathrm{i}}^{\mathrm{n}}\right) \\
& =\frac{\gamma_{\mathrm{o}} \rho_{0}}{1+\gamma_{\mathrm{o}} \eta_{\mathrm{i}}^{\mathrm{n}}} \mathrm{e}_{\mathrm{i}}^{\mathrm{n}}+\rho_{0} \mathrm{c}_{0}^{2} \eta_{i}^{\mathrm{n}} \frac{2-\gamma_{\mathrm{o}} \eta_{i}^{\mathrm{n}}}{2\left(1+\gamma_{\mathrm{o}} \eta_{i}^{\mathrm{n}}\right)\left(1-s \eta_{i}^{\mathrm{n}}\right)^{2}} \\
& \eta_{i}^{\mathrm{n}}=1-\frac{\rho_{0}}{\rho_{i}^{\mathrm{n}}}
\end{aligned}
$$

With

$\rho_{i}^{0}=\rho_{0}, w_{i}^{0}=\delta\left(x_{i}\right) \rho_{0}$

$\mathrm{E}_{\mathrm{i}}^{0}=\rho_{0} \mathrm{e}_{0}+\frac{1}{2}\left(\mathrm{w}_{\mathrm{i}}^{0}\right)^{2} / \rho_{0}$.

Note that for the first time interval only (10)-(11) and (14)-(15) are used with $\tau$ set to zero. At the end of the first time interval the full system is used.

Once $\rho, w$ and $E$ are obtained the relations $v=w / \rho$ and $e=E / \rho-v^{2} / 2$ are used to get $v$ and $e$.

For the first time interval the system (9) can be written in the matrix form $U_{t}=-F_{X}(U)$ where $U=\left(\begin{array}{c}\rho \\ w\end{array}\right)$ and $F=\left(\begin{array}{c}w \\ \frac{w^{2}}{\rho}+p\end{array}\right)$. It is well know that the Lax Wendroff scheme is stable if the CFL condition, $\frac{k}{h} \sup (|\lambda|) \leq 1$ is satisfied. Here $\lambda$ is an eigenvalue of the Jacobian matrix $\partial \mathrm{F} / \partial \mathrm{U}$ of $\mathrm{F}$. We have

$$
\partial \mathrm{F} / \partial \mathrm{U}=\left(\begin{array}{cc}
0 & 1 \\
-\frac{\mathrm{w}^{2}}{\rho^{2}}+\mathrm{a} & \frac{2 \mathrm{w}}{\rho}
\end{array}\right)
$$

Where $\mathrm{a}=\partial \mathrm{p} / \partial \rho$. The eigenvalues of (19) are $\lambda_{1}=\frac{1}{\rho}(\mathrm{w}+\sqrt{\mathrm{a} \rho})$, $\lambda_{2}=\frac{1}{\rho}(w-\sqrt{a} \rho)$. So, for the first time intervals the method is stable if $\frac{\mathrm{k}}{\mathrm{h}} \leq \frac{1}{\mathrm{v}+\sqrt{\mathrm{a}}}$, assuming that $\mathrm{w}$ and $\rho$ are positive.

For the second time interval we have

$\partial \mathrm{F} / \partial \mathrm{U}=\left(\begin{array}{ccc}0 & 1 & 0 \\ -\frac{\mathrm{w}^{2}}{\rho^{2}}+\mathrm{a} & \frac{2 \mathrm{w}}{\rho}+\mathrm{b} & \mathrm{c} \\ -\frac{\mathrm{w}}{\rho^{2}}(\mathrm{E}+\mathrm{p})+\frac{\mathrm{w}}{\rho} \mathrm{a} & \frac{1}{\rho}(\mathrm{E}+\mathrm{p}) & \frac{\mathrm{w}}{\rho}(1+\mathrm{c})\end{array}\right)$

Where $b=\partial \tau / \partial w$ and $c=\partial p / \partial E$. If $|D|$ is small then $b$ can be neglected and the eigenvalues in this case are given by

$$
\begin{aligned}
& \lambda_{1}=\frac{1}{2 \rho}\left(2 \mathrm{w}-\sqrt{4 \mathrm{a}^{2}+\mathrm{c}^{2} \mathrm{w}^{2}+4 \mathrm{cp \rho}+4 \mathrm{c} \rho \mathrm{E}}+\mathrm{cw}\right) \\
& \lambda_{2}=\frac{1}{2 \rho}\left(2 \mathrm{w}+\sqrt{4 \mathrm{a}^{2}+\mathrm{c}^{2} \mathrm{w}^{2}+4 \mathrm{cp} \rho+4 \mathrm{c} \rho \mathrm{E}}+\mathrm{cw}\right) \\
& \lambda_{3}=\frac{\mathrm{w}}{\rho}
\end{aligned}
$$

So, assuming that $\rho$ and $\mathrm{w}$ are positive, the method is stable if

$\frac{\mathrm{k}}{\mathrm{h}} \frac{1}{2 \rho}\left(2 \mathrm{w}+\sqrt{4 \mathrm{a} \rho^{2}+\mathrm{c}^{2} \mathrm{w}^{2}+4 \mathrm{cp \rho}+4 \mathrm{c} \rho \mathrm{E}}+\mathrm{cw}\right) \leq 1$

\section{Numerical results}

Empirical Mie-Gruneisen constants are shown in Table 2 for three types of sandy media: 
Table 2: Mie-Gruneisen Constants

Dry-Silica Sand - Porosity 41\%

\begin{tabular}{|c|c|c|}
\hline$\gamma_{0}=13.8509$ & $\mathrm{~s}=1.6369$ & $\mathrm{c}_{0}=0.0606 \mathrm{~cm} / \mu \mathrm{sec}$ \\
\hline \multicolumn{3}{|c|}{ Dry-Silica Sand - Porosity 22\% } \\
\hline$\gamma_{0}=14.8726$ & $\mathrm{~s}=2.1451$ & $\mathrm{c}_{0}=0.0442 \mathrm{~cm} / \mu \mathrm{sec}$ \\
\hline $\begin{array}{c}\text { Water-Saturated S } \\
\gamma_{0}=5.2676 \\
\end{array}$ & $\begin{array}{r}\text { a Sand - Porosity } \\
\mathrm{s}=1.5406 \\
\end{array}$ & $\mathrm{c}_{0}=0.269 \mathrm{~cm} / \mu \mathrm{sec}$ \\
\hline
\end{tabular}

Given the values of the Mie-Gruneisen constants, $\gamma_{0}$, s, and $\mathrm{c}_{0}$, with the empirical constants in the deviatoric stress tensor $\varepsilon=10^{-3}, \mathrm{n}=10$ and $\mu=10^{-4}$, we now look at the outward of the speed of propagation for the shock waves $w$ given by

$$
\mathrm{w}(\mathrm{x}, \mathrm{t})]^{2}=\partial \mathrm{p} / \partial \rho
$$

The Lax-Wendroff scheme was used to determine values for the speed of the shock waves at various distances from the point of explosion. We will compare the results given when the deviatoric tensor both included and not included in the system of equation in order to study the behavior of the speed waves.

The following initial and boundary conditions given by Eqs. (4)(5) will be used for the numerical solution:

$\rho(x, 0)=\rho_{0}=1.98 \mathrm{~g} / \mathrm{cm}^{3}$,

$\mathrm{v}(0,0)=\mathrm{V}_{0}=0.04572 \mathrm{~cm} / \mu$ sec and $\mathrm{v}(\mathrm{x}, 0)=0$ for $\mathrm{x}>0$

$\mathrm{e}(\mathrm{x}, 0)=\mathrm{e}_{0}=222775.2817 \mathrm{~J} / \mathrm{kg}=0.00228 \mathrm{~cm}^{2} / \mu_{\mathrm{sec}^{2}}$

The spatial step $\mathrm{h}$ is taken as small as possible to allow the disturbance to be close to $\mathrm{x}=0$, but must also be large enough in proportion to the time step size $\mathrm{k}$ to allow the numerical scheme to be stable.

Figs. 2-7 show the speed of the shock waves for three different types of silica sand listed in Table 2. Figs. 2-3 show the speed of the shock waves for the wet sand with porosity $41 \%$ in the first and second time intervals respectively. Similarly Figs. 4-5 and 6-7 show the speed of the shock waves for dry sands with porosity $41 \%$ and $22 \%$, respectively, in both time intervals.

From the graphs of the numerical results one can observe that the speed of the shock wave is faster in the wet sand than the dry sands (see [1]). In the contrary, the amplitude of the shock wave in the wet sand is approximately one-third of the one in the dry sands and it damps faster in the wet than in the dry sands. In addition, one can observe a net decrease of the amplitude of the shock waves in all three types of sand after the first time interval. That is, after the first 1000 micosec.

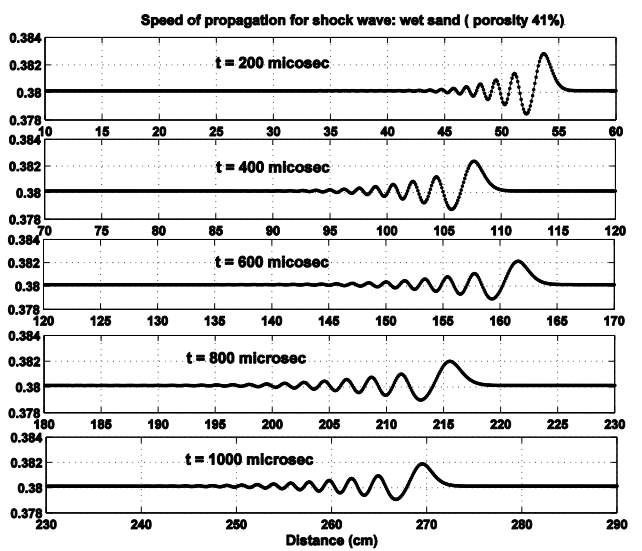

Fig. 2: First Time Interval.

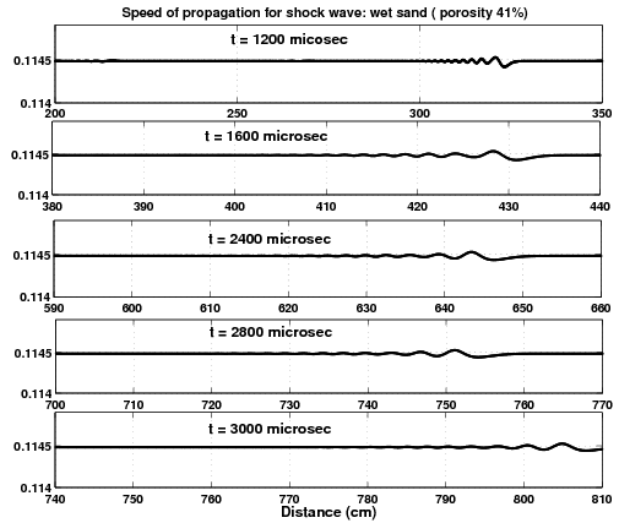

Fig. 3: Second Time Interval.

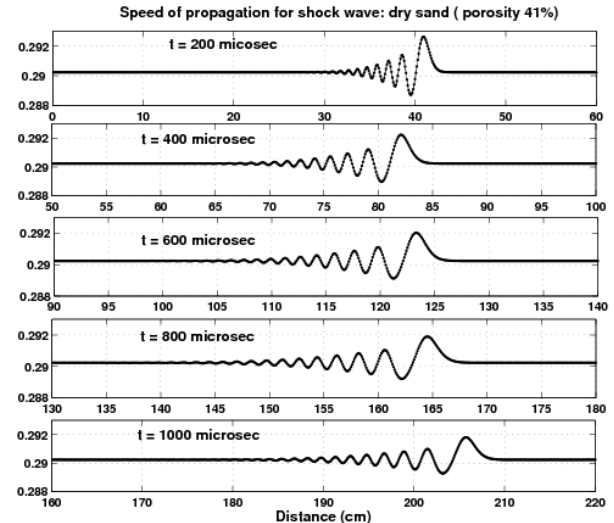

Fig. 4: First Time Interval.

Speed of propagation for shock wave: dry sand ( porosity $41 \%$ )
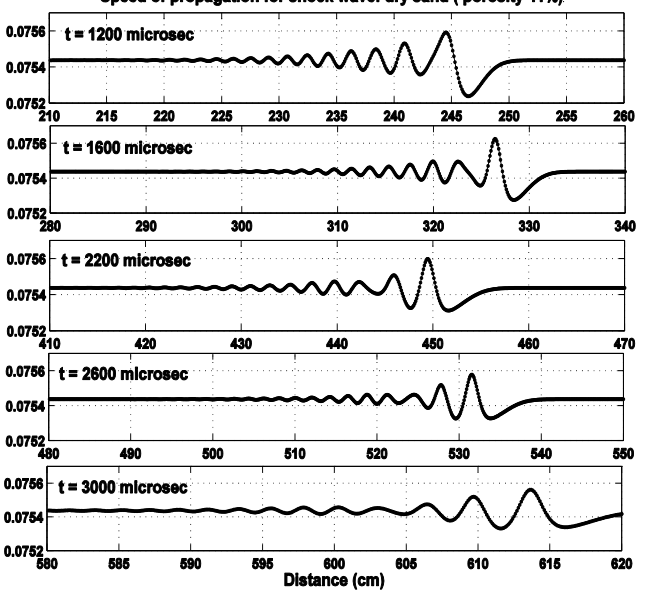

Fig. 5: Second Time Interval.

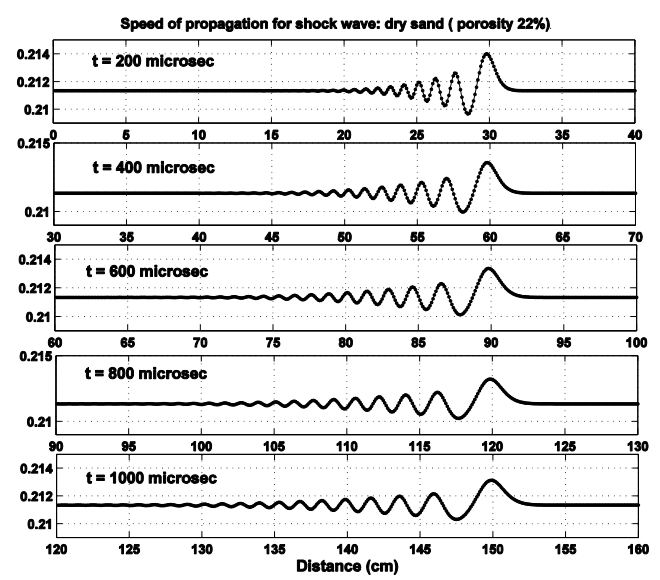

Fig. 6: First Time Interval 


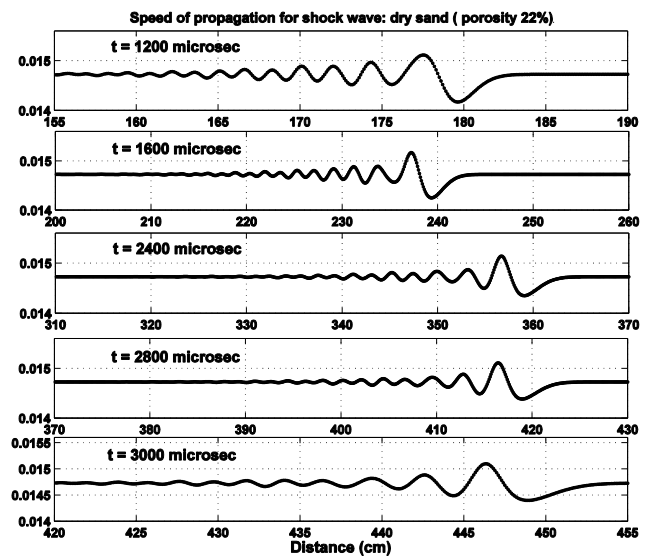

Fig. 7: Second Time Interval.

\section{Conclusion}

This work addressed the numerical investigation of an explosion or a penetration of a typical rigid body into a sand medium. Conservative laws in continuum mechanics were used to model the problem. Numerical results were obtained using the LaxWendroff scheme to analyze the speed of the shock waves for three types of sandy media. Further study that uses different forms of the stress-strain relation and different type of soil could be conducted for a better analysis of soil explosion or penetration problems.

\section{Acknowledgement}

The authors wish to acknowledge the financial support of Abu Dhabi University, Abu Dhabi.

\section{References}

[1] M. Arley and al., Dynamic behavior of dry and water-saturated sand under plane shock conditions, Int. J. of Impact Eng., 37 (2010) 1-10. https://doi.org/10.1016/j.ijimpeng.2009.07.009.

[2] M.E. Backman, W. Goldsmith, The mechanics of penetration of projectiles into targets, Int. J. Engng Sci., 16 (1978) 1-99. https://doi.org/10.1016/0020-7225(78)90002-2.

[3] M.J. Forrestal, K. Okajima, V.K. Luk, Penetration of 6061-T651 aluminum targets with rigid long rods, ASME J. of Appl. Mech., 55 (1988) 755-60. https://doi.org/10.1115/1.3173718.

[4] M.J. Forrestal, A.J. Piekutovvski, Penetration experiments with 6061-T651 aluminum targets and spherical-nose steel projectiles at striking velocities between 0.5 and $3.0 \mathrm{~km} / \mathrm{s}$, Int. J. Impact Eng., 24 (2000) 57-67. https://doi.org/10.1016/S0734-743X(99)00033-0.

[5] X.W. Chen, Q.M. Li, Deep penetration of a non-deformable projectile with different geometrical characteristics, Int. J. Impact Engng., 27(6) (2002) 619-37. https://doi.org/10.1016/S0734743X(02)00005-2.

[6] W. Goldsmith, Impact. The theory and physical behavior of colliding soils, Dover Publications Inc. Mineda, New York, 2001.

[7] A. Kharab, R.T. Hudspeth, R.B. Guenther,Penetration of Cylindrical Projectiles into Saturated Sandy Media, Experimental Mech., 49 (2009) 605-612. https://doi.org/10.1007/s11340-008-9190-9.

[8] T.C. Papanastasiou, A.G. Boudouvis, Flows of viscous materials: Models and computations, Computers \& Structures, 64 (1997) 677 694. https://doi.org/10.1016/S0045-7949(96)00167-8.

[9] A.L. Yarin, Rubm M.B., Roisman I.V., Penetration of a rigid projectile into an elastic-plastic target of finite thickness, Int. J. Impact Engng., 16 (1995) 801-31. $\quad$ https://doi.org/10.1016/0734743X(95)00019-7.

[10] I.V. Roisman , A.L.Yarin, M.B. Rubin, Oblique penetration of a rigid projectile into an elastic-plastic target, Int. J. Impact Engng., 19 (1997) 769-95. https://doi.org/10.1016/S0734-743X(97)00014-6.

[11] T.L. Warren, M.J. Forrestal, Effects of strain hardening and strainrate sensitivity on the peikii of aluminum targets with sphericalnose rods, Int. J. Solids Struct., 35 (1998) 3737-52. https://doi.org/10.1016/S0020-7683(97)00211-4. 